Conclusions: The factors that are in direct relations with exacerbation of MS in our study are: season, residence and age.

10

\section{Intrathecal IgM synthesis in children with multiple sclerosis is associated with a slower progression} C. Stauch $M D^{1}$, M. Rauchenzauner $M^{3}{ }^{3}$, D. Pohl MD ${ }^{4}$, F. Hanefeld MD ${ }^{1}$, H. Reiber ${ }^{2}$ \& K.M. Rostásy ${ }^{3}$

${ }^{1}$ Department of Pediatrics and Pediatric Neurology, Georg-August

University Göttingen, Germany, ${ }^{2}$ Neurochemistry Laboratory,

Department of Neurology, Georg-August University Göttingen,

Germany, ${ }^{3}$ Department of Pediatrics IV, Division of Pediatric Neurology

and Inborn Errors of Metabolism, Medical University of Innsbruck,

Austria, ${ }^{4}$ Department of Neurology, Childrens Hospital of Eastern

Ontario, Ottawa, Ontario, Canada

E-mail: Kevin.Rostasy@uki.at

Background: Intrathecal IgM synthesis has been associated with the onset of new relapses and an earlier onset of secondary progressive disease in adult multiple sclerosis patients.

Objective: Investigation of the predictive value of intrathecal IgM by correlation of recent interpretations of CSF data with clinical information from pediatric MS patients.

Methods: Seventy-two children with onset of MS before age of 16y were followed for a mean period of 10.3 years (range: $0.4-22.8$ years) evaluated as two groups with $(n=44)$ or without intrathecal IgM synthesis $(n=28)$. Clinical course and EDSS scores at five and 10 years were compared with CSF data interpreted with a non-linear program for statistics of groups in $\mathrm{CSF} /$ serum quotient diagrams.

Results: In general, female gender, total number of attacks, number of attacks in the first 2 years and the time interval between first and second attack were associated with a worse prognosis. The cohort of children without intrathecal $\operatorname{IgM}$ had a significant higher number of relapses in the first 2 years $(P=0.033)$ with a trend to shorter time intervals between first and second attack and a higher EDSS score after 10 years of MS, though not statistically significant. In the subgroup of girls without intrathecal IgM EDSS score after 10 years was significantly higher compared to the group with IgM synthesis $(P=0.023)$. The contradiction to earlier reports is explained as a bias in the qualitative method or interpretation with a linear IgM Index. Conclusion: Intrathecal IgM synthesis at time of first clinical manifestation was associated with a slower progression of disability in pediatric MS.

\section{1}

\section{The role of conventional MR imaging in diagnosing multiple sclerosis}

Pećina Hrvoje, Šverko Ana, Bedek Darko, Podoreški Dijana, Gregurić Tomislav \& Hat Josip

Institute for Diagnostic and Interventional Radiology, University Hospital Sestre Milosrdnice, Department of Interventional and Diagnostic

Radiology "Sestre Milosrdnice" University Hospital, Zagreb, Croatia E-mail: hrvoje.pecina@zg.t-com.hr

Conventional MR as an important paraclinical tool for diagnosing multiple sclerosis (MS) and monitoring therapeutic trials offers by far the most sensitive technique for detecting MS lesions.

Its purpose is especially important in ruling in or ruling out a diagnosis of MS. The ability to detect lesions depends on the pulse sequence, imaging parameters and the field strength.

There is a special MR protocol which has to be performed with application of gadolinium contrast media in order to increase the sensitivity and specificity of conventional MR examination since the lesion number and location are included in the diagnostic criteria of MS.

Although MS lesion plaques can be found throughout the brain, spinal medulla, orbit and cranial nerves, they usually have specific features which help to distinguish MS from other demyelinating, cerebrovascular diseases or any other diseases that affect white matter of the brain. Conventional MR is also helpful in distinguishing MS variants and subtypes.

Although conventional MR is a powerful paraclinical technique to depict MS lesions, one should bear in mind that there are cases of MR-negative MS. Therefore, to obtain the diagnosis of MS it is necessary to synthesize the results of clinical exams, laboratory tests and all the paraclinical exams as well as their changes in time.

12

\section{Similarity and difference among multiple sclerosis group during psychiatric controls}

Eduard Pavlović, Mirjana Janković \& Marija Vučić Peitl

Psychiatric Clinic, Clinic Hospital Center, Cambierieva 17/7, 51000

Rijeka, Croatia

E-mail: edopav@excite.com

Introduction/Objectives: The main of this research was to show what kind of sociodemographic and clinical differences were among the multiple sclerosis during psychiatric controls.

Participants, Materials/Methods: Every of age patient with multiple sclerosis (MS) who was control by psychiatrists at Polyclinic of CHC Rijeka during Mart 2009 was included in this study. The wide sociodemographic and clinic facts were registered in the polyclinical patients card boards.

Results: During Mart 2009 there were 8 patients (m-3, f-5) on their regular controls. Six or $75 \%$ of them were more than 40 year old. In 5 or in $62.5 \%$ of them MS were diagnosed later. About $87 \%$ of them worked longer than 20 years and they were on sick leaves longer than 10 years but 6 or $75 \%$ of them contacted with irritant materials on their work places. Mainly of them (6 or $75 \%$ ) suffered from depression. Only one of them was born in Gorski kotar (the part of Croatia with high incidence for MS) and only one of them had the positive familiar anamnesis according to MS.

Conclusions: The persons with MS often suffered from depression. About $75 \%$ of them contacted with irritant materials during their long work time.

\section{3}

\section{Magnetic resonance of white matter lesions}

Šverko Ana, Pedišić Ivo, Bedek Darko, Gregurić Tomislav, Pećina

Hrvoje, Podoreški Dijana, Borojević Nikola \& Hat Josip

Institute for Diagnostic and Interventional Radiology, University

Hospital Sestre Milosrdnice, Zagreb, Croatia

E-mail: ana.sverko@gmail.com

Introduction: Differential diagnosis of white matter lesions (WML) is quite extensive and includes hypoxic-ischemic origin, inflammation, infection, toxic or metabolic agents, trauma, hereditary diseases or even normal aging. One of the most common questions to be answered is: do the lesions represent multiple sclerosis? At the moment there is no single MRI technique that could unambiguously answer this question.

The aim of the study is to get acquainted with possibilities of conventional magnetic resonance imaging (MRI) in detection, characterization and differentiation of white matter lesions.

Materials and methods: $1 \mathrm{~T}$ or $1.5 \mathrm{~T}$ MRI scanner were used to examine the patients suspected for WML and images were acquired according to the standard protocol at our institution. 
Results: In selected illustrative cases we present imaging findings characteristic for different pathological white matter processes.

Conclusion: MRI is a very sensitive but unfortunately relatively unspecific method for evaluation of WML. As mentioned earlier, differential diagnosis of WML is a long list. Due to that the diagnosis of a specific pathological process characterised by one or more lesions in the white matter has to be made by combining clinical aspect and radiological assessment.

\section{4}

\section{Surveillance of Croatian pregnant women with epilepsy and effects of antiepileptic drugs exposure in their offspring}

Snježana Miškov ${ }^{1}$, Romana Gjergja Juraški ${ }^{2}$, Aleksandra Fučić ${ }^{3}$, Tomislav Ivičević Bakuli ${ }^{4}$, Ljerka Cvitanović Šojat ${ }^{2}$, Vlasta Vuković ${ }^{1}$, Vanja Basic Kes ${ }^{1} \&$ Vida Demarin ${ }^{1}$

'Department of Neurology, University Hospital "Sisters of Mercy", Zagreb, Croatia, ${ }^{2}$ Department of Pediatrics, University Hospital "Sisters of Mercy", Vinogradska cesta 29, Zagreb, Croatia, ${ }^{3}$ Institute for Medical Research and Occupational Health, Ksaverska c 2, Zagreb, Croatia, ${ }^{4}$ Department of Obstetrics and Gynecology, University Hospital "Sisters of Mercy", Vinogradska cesta 29, Zagreb, Croatia

Introduction: The aim was to survey pregnancies exposed to AED and their offspring in order to assess teratogenic/ neurodevelopmental effect of newer generation AEDs.

Methods: This is prospective surveillance of pregnancies in women with epilepsy(May2003-May2008). Pregnancy planning data, folic acid (FA) supplementation, seizure frequency and AED therapy were obtained.

Results: From 47 pregnancies: $83 \% \quad(39 / 47)$ exposed to monotherapy: 23 to lamotrigine (LTG): 13 live-births (LB), 2 premature deliveries, 2 spontaneous abortions (SA), 1 artificial abortion, 1 intrauterine death and 4 ongoing pregnancies (OP). Seven LB were exposed to carbamazepine (CBZ), 1 LB was under phenitoine (PHT) and 1 under phenobarbiton (PB) with EPH gestosis/peripartal asphyxia. One preterm LB (ASD), severe psychomotor delay and epilepsy) was exposed to gabapentine (GBP), 3LB and 1 OP were under valproic acid (VP). One LB and 1 $\mathrm{SA}$ were under phenobarbiton (PB). Six pregnancies were exposed to polytherapy: topiramate (TPM)/VP (1 LB, $1 \mathrm{SA}, 1 \mathrm{OP}) \mathrm{CBZ} / \mathrm{PB}$ (1stillbirth); TPM/CBZ/PHT (1LB) with intrauterine growth retardation and dysmorphism); $\mathrm{VP} /$ clonazepam (CZP) (1 OP). Two women without AED therapy delivered healthy LB. From $35 \%$ planned pregnancies, $20 \%$ took FA properly. About $25.5 \%$ of these women had their second or third pregnancy during our survey and their pregnancy planning was above $50 \%$ mainly due to preconceptional counseling.

Conclusion: Pregnancies under polytherapy resulted in larger proportion of complications. Besides 4 SA, 2 still-births, 2 premature deliveries, we have noted 1 possible intrauterine AED effect and 1 premature LB with ASD, psychomotor delay and epilepsy. Adequate preconceptional counseling in women with epilepsy resulted in higher pregnancy planning and FA intake. Follow up of LB till school age is needed.

\section{5}

\section{Public knowledge and predictors for negative attitudes toward patients with epilepsy in Croatia}

Hrvoje Hećimović, Snježana Miškov, Jelena Bošnjak, Ružica Negovetić \& Vida Demarin

Zagreb Epilepsy Center, Department of Neurology, University Hospital, Zagreb, Croatia
Rationale: We assessed public knowledge of and attitudes toward people with epilepsy in the Croatian population. We also aimed to identify independent predictors of negative attitudes.

Methods: We performed a survey consisting of a representative sample of 1500 adults. The population tested were 18 years or older, living in a private households. Croatia is a country of 5.5 million inhabitants with a socioeconomic and ethnic profile similar to neighboring Middle European countries. Within the sample, the respondents were selected according to the random route method that guaranteed a representative sample concerning households. The survey consisted of questions intended to determine the respondents' socioeconomic background and education, acquaintance with persons with epilepsy and knowledge and attitudes towards them. Urban and rural population was tested living in the places of less than 2.000 to more than 100.000 inhabitants. This survey consisted of 14 questions, tailored to the ones used in earlier studies of public knowledge towards people with epilepsy in other countries. Logistic regression analysis was used to test for independent predictors of negative attitudes. All data were stored and analyzed in the SPSS database.

Results: We surveyed a representative sample of Croatian population aged between 18-94 years. Majority were females $(55.3 \%)$, living in urban setting $(61.3 \%)$ with high school or higher degree $(78.6 \%)$. Majority of the respondents worked for a public or private company $(37.5 \%)$, and $34 \%$ were retired.

The results show that $91 \%$ of respondents have heard about epilepsy, $57 \%$ of them know a person with epilepsy and $55 \%$ have witnessed a seizure. $48 \%$ believed that people with epilepsy are treated differently in the society. Majority of them presume that epilepsy is a mental illness ( $41 \%), 29 \%$ that people with epilepsy should have a lower quality job, $17 \%$ do not want that their son/ daughter live with person with epilepsy, $17 \%$ think that people with epilepsy should not have children, and $12 \%$ that they should attend special schools. $87 \%$ of respondents without any regular education assume that people with epilepsy should have a lower quality job, however, this is still high belief in the people having a university degree $(25 \%)$. Consistent independent predictors for all attitudes were a positive response to the question whether one has heard or read about epilepsy, the misconception of epilepsy as a form of mental illness and an infectious disorder. Employment and knowing someone with epilepsy were independent predictors for four out of five tested attitudes.

Conclusion: This is the first study assessing public knowledge of and attitudes toward people with epilepsy in the Croatian population. We showed that public knowledge of epilepsy is similar to other countries, and we defined predictors for negative attitudes. Future educational campaigns on epilepsy should improve attitudes toward epilepsy mainly by targeting misconceptions about epilepsy and by offering opportunities for personal acquaintance with people with epilepsy.

16

\section{Importance of early recognition of adverse drug reactions (ADR) induced by lamotrigine}

Banovac Marin, Mirošević Nikica, Macolić Šarinić Viola, Arapović Selma \& Tomić Siniša

Pharmacovigilance Department, Agency for Medicinal Products and Medical Devices, Ksaverska Cesta 4, 10000 Zagreb, Croatia

E-mail: marin.banovac@almp.hr

Introduction/Objectives: During the period from March 2005 to April 2008 Croatian Agency for Medicinal Products and Medical Devices (Agency) received 10 reports of ADRs associated with lamotrigine. Three of those were severe ADRs including Stevens Johnson syndrome and hypersensitivity including multi-systemic 